\title{
Project for the prevention of caries in the developmental age: Experience in kindergartens of Northern Italy
}

\section{Abstract}

Introduction: Caries is one of the most widespread chronic diseases in the world and in Italy, only $78.4 \%$ of the 4 years children are caries free. Caries is defined as an infectious multifactorial pathology and between the etiological factors that contribute to its developing we find excessive sugar consumption. Correct behaviours adopted since the pediatric age will allow the child to learn how to improve their lifestyle, thus protecting health. Parents must therefore be properly educated to transmit healthy habits to their children from the early years. The main goal of our project is the prevention of caries in the developmental age by means of educational interventions on parents of children in the kindergarten (0-3 years). The study assesses the oral hygiene and food habits of the child and caregiver at baseline and after 3 months from the interventions (follow-up).

Materials and Methods: We have used a questionnaire to collect the oral hygiene and food habits of parents and children. Next, dental hygienists and nutritionists organized training sessions for parents about correct oral hygiene and eating habits (in particular to reduce the sugar consumption). After 3 months from the training, the same questionnaires reconfirmed to evaluate the improvement of family habits.

Results: The number of participants was reduce from 99 to 68 at the follow-up. With regards to eating habits, after training, almost the whole of the sample began to practice breakfast, indicating that the actual importance of this meal was understood. The habit of morning and afternoon snack, essential for a growing child, has increased (from $84 \%$ at baseline to $94 \%$ at follow-up and from $92 \%$ to $97 \%$ respectively). The bad habit of the after dinner snack, often even after teeth brushing, has decreased consistently $(32 \%$ to $22 \%)$.

The most significant advances in oral hygiene are those concerning tooth washing ( $86 \%$ to $97 \%$ ), the average daily washing (1.27/die to $1.57 /$ die), self-washing ( $33 \%$ to $30 \%)$, the use of toothpaste $(85 \%$ to $90 \%)$ and fluoridated toothpaste $(48 \%$ to $62 \%)$.

Discussion: Starting from the data obtained from our pilot study, we think that it is essential to intervene already in the first years of life, when the subject is still "plastic" and can be trained to perform correct eating habits and oral hygiene. Furthermore, reducing the consumption of foods rich in sugar does not have a positive effect only on the onset of caries but also on the health of the individual.

\section{Conflict of Interest}

there is no conflict of interest 